American Journal of Infectious Diseases 8 (2): 92-105, 2012

ISSN 1553-6203

(C) 2012 Science Publications

\title{
Monocytes and their Role in Human Immunodeficiency Virus Pathogenesis
}

\author{
Tara Sassé, Jingqin Wu, Li Zhou and Nitin K. Saksena \\ Retroviral Genetics Division, Center for Virus Research, \\ Westmead Millennium Institute, Westmead Hospital, \\ University of Sydney Westmead, Sydney, NSW Australia
}

\begin{abstract}
Monocytes play several significant immunological roles during HIV infection. The phenotypic pliability and the cellular differentiation ability monocytes possess are crucial to the ways they combat infections and control inflammatory processes. The purpose of this review is to provide a comprehensive snapshot of the importance of monocytes in HIV-1 infection and pathogenesis. Moreover, this review also provides newly emerging data on how HIV leads to the subversion and manipulation of monocyte transcriptome and proteome, which may have implications in understanding the genomic and proteomic basis of monocyte function and its interaction with HIV.
\end{abstract}

Key words: Dendritic Cells (DCs), Human Immunodeficiency Virus (HIV), Tumor Necrosis Factor (TNF), Natural Killer (NK), Lipopolysaccharide (LPS)

\section{INTRODUCTION}

According to the UNAIDS estimate, 34 million people around the world were living with HIV at the end of $2010 ; 17 \%$ more people living with HIV than in 2001. It is estimated that 2.7 million people became infected in 2010, including close to 400000 children. This means that AIDS remains one of the most significant infectious diseases worldwide and these figures are not likely to decrease, with over 7300 people becoming infected with HIV every day (UNAIDS/WHO, 2010).

The causative agent of AIDS was first reported in 1983 (Barre-Sinoussi et al., 1983), which was named Human Immunodeficiency Virus (HIV) (Coffin et al., 1986). HIV has two major sub-species: the more common HIV type 1 (HIV-1) and the less prominent HIV type 2 (HIV-2), which was discovered in West Africa in 1986 (Clavel et al., 1986). HIV-2 can also cause AIDS (Rowland-Jones and Whittle, 2007), but it has remained localized to west Africa and the reasons for its very low infectious potential and inefficient global spread remain unexplained. In contrast, HIV-1 has successfully spread across the globe and is responsible for the current global AIDS pandemic. Although HIV infects primarily the CD4+T cells, it has the ability to infect all major blood leukocytes, including monocytes (Saksena and Potter, 2003).
Monocytic phenotypes in vivo: There are two types of monocytes in the human blood: (a) the classical monocyte, which is characterized by high level expression of the CD14 cell surface receptor (CD14++monocyte) and (b) the non-classical, proinflammatory monocyte with low level expression of CD14 and with additional co-expression of the CD16 receptor (CD14+CD16+monocyte) (Ziegler-Heitbrock, 2007). The CD14+CD16+monocytes develop from the CD14++monocytes, i.e., they are a more mature version. Following stimulation with microbial products the CD14+CD16+monocytes produce copious amounts of pro-inflammatory cytokines like Tumor Necrosis Factor (TNF) and interleukin-12 (IL-12).

Functional attributes of Monocytes: In humans, about $3-8 \%$ of blood leukocytes is comprised of monocytes and is produced by the bone marrow from monoblaststhe haematopoietic stem cell precursors. Recently, scientists from the Massachusetts General Hospital and Harvard Medical School demonstrated that the spleen is a reservoir for huge numbers of monocytes. A current paradigm states that monocytes circulate freely and patrol blood vessels but irreversibly differentiate into Dendritic Cells (DCs) or macrophages upon tissue entry. They show clear evidence that undifferentiated monocytes reside in the spleen and outnumber their equivalents in systemic circulation. Normally, monocytes are known to systemically circulate for

Corresponding Author: Nitin K. Saksena, Retroviral Genetics Division, Center for Virus Research, Westmead Millennium Institute, Westmead NSW 2145, Sydney, Australia 
Am. J. Infect. Dis., 8 (2): 92-105, 2012

about one to three days before moving into tissues throughout the body. The reservoir monocytes assemble in clusters in the cords of the sub-capsular red pulp and are distinct from macrophages and DCs. These observations have uncovered a role for the spleen as a site for storage and rapid deployment of monocytes and identify splenic monocytes as a resource that the body exploits to regulate inflammatory processes (Swirski et al., 2009). These observations have created a new paradigm that will significantly shift the way we perceive monocytes in the context of HIV infection and their deployment and sequestration to inflammation sites in vivo.

Monocytes play several significant roles in the immune system; (1) they replenish resident macrophages and dendritic cells under normal states, (2) they, in response to inflammation signals, move quickly (approx. 8-12 h.) to sites of infection in the tissues and divide/differentiate into macrophages and dendritic cells to elicit an immune response and (3) they can perform phagocytosis using intermediary (opsonising) proteins such as antibodies or complement that coat the pathogen, as well as by binding to the microbe directly via pattern-recognition receptors that recognize pathogens. They are also capable of killing infected host cells via antibody, termed antibodymediated cellular cytotoxicity. Thus, the blood monocytes are young cells that already possess migratory, chemotactic, pinocytic and phagocytic activities, as well as receptors for IgG Fc-domains (FcR) and iC3b complement. Upon migration into tissues, monocytes undergo further differentiation to become multifunctional tissue macrophages. Monocytes, therefore, are considered to be immature macrophages. It can also be argued that monocytes represent the circulating macrophage population and therefore should be considered fully functional for their location as they can change their phenotype in response to factors encountered in specific tissue post-migration.

\section{HIV infection of diverse blood leukocytes including Monocytes: \\ HIV infection of blood leukocytes: HIV is capable of} infecting cells of diverse types. A wide-range of cell types distributed amongst a number of different tissues is susceptible to HIV infection. CD4+T lymphocytes (Klatzmann et al., 1984; Schnittman et al., 1989) and macrophages (Gartner et al., 1986; Ho et al., 1986) are the primary cellular targets of HIV. Other infectable cell types include monocytes, CD8+T lymphocytes, Natural Killer (NK) cells, dendritic cells, B cells and an array of specialized cell types derived from various tissue reservoirs of HIV (e.g., renal, mucosal and cervical epithelial cells, astrocytes and microglia in the
CNS, skin fibroblasts, bone marrow stem cells). However, the infection of a number of putative cellular HIV targets remains controversial and therefore the actual estimate of their contribution to HIV pathogenesis is often unclear.

HIV-1 can infect cells by two mechanisms, either by the attachment of cell-free virions, or by the direct passage of HIV-1 between infected and uninfected cells. HIV usually disseminates by a direct cell-cell transfer mechanism in densely populated tissues, such as between $\mathrm{T}$ lymphocytes in the lymph nodes. Jolly $e t$ al. (2004) showed that this direct passage of HIV-1 is centered on an adhesive junction, where by HIV-1 virions can move through and infect the target cell. This adhesive junction contains CD4 and chemokine receptors, as well as adhesion molecules suchas LFA-1 and ICAM-1. These receptors are up-regulated and migrate to the synapse via an actin-dependent mechanism (Jolly et al., 2004). This up-regulation and migration of receptors is probably initiated by the attachment of initial Env glycoproteins to CD4 and the chemokine receptor, which in turn induces the recruitment of more receptors.

Monocytes: In the context of HIV, the infection of circulating blood monocytes was first reported early in the epidemic (Gartner et al., 1986; Ho et al., 1986; (McElrath et al., 1989; 1991). These initial reports did not clarify as to whether monocyte infection is latent or productive. Subsequent studies found levels of proviral DNA in monocytes to be relatively low or undetectable in comparison to T cell compartments (Innocenti et al., 1992; Livingstone et al., 1996). However, more sophisticated approaches using in situ hybridization coupled with simultaneous surface immuno-phenotyping revealed a higher incidence of monocyte infection and demonstrated the production of viral mRNA in monocytes indicating productive infection (Patterson et al., 1993; 1995; 1999). Despite the apparent production of viral mRNA, other in vitro studies indicated that HIV replication was blocked prior to reverse transcription and integration (Sonza et al., 1996). Following this, replication competent virus was shown to be recoverable from blood-derived monocytes upon stimulation and differentiation into macrophages (Lambotte et al., 2000). But one is to be cautious as other contaminant cell types with monocytes can lead to misinterpretation of actual infection of monocytes by HIV.

The HIV envelope protein gp120 must interact with both CD4 receptor and a co-receptor to trigger fusion of the viral and cellular membranes and gain entry into the target cells. The ability to bind to specific co-receptors is a critical determinant of the cell tropism 
Am. J. Infect. Dis., 8 (2): 92-105, 2012

of different HIV-1 strains. For example, binding to the $\alpha$-chemokine receptor CXCR4 is a definitive feature of strains that infect $\mathrm{T}$-cell lines, where as binding to the $\beta$-chemokine receptor CCR5 is characteristic of Mtropic strains, which primarily infect monocytes and macrophages. There is direct association between levels of CCR5 and differentiation of monocytes to macrophages. Levels of CCR5 are related to monocyte resistance and macrophage susceptibility to infection because the infection by the M-tropic strain HIV-1JRFL could be blocked by MAb 2D7. This direct evidence suggests that CCR5 functions as a co-receptor for HIV-1 infection of primary macrophages (Tuttle et al., 1998). Further, it has also been shown that the CCR5 expression correlates with the susceptibility of maturing monocytes to HIV-1 infection (Naif et al., 1998).

Monocytes express both CD4 receptor and CCR5 and CXCR4 co-receptors, so HIV-1 viral entry into monocytes is possible (Joly and Pinto, 2005). Therefore, the control of viral infection in monocytes is thought to take place during the early phases of infection, after viral entry. Arfi et al. (2008) showed that monocytes are susceptible to HIV-1, but the cells display several defects during HIV-1 infection, such as a slow reverse transcription and delayed nuclear import and integration (Arfi et al., 2008). Synthesis of viral DNA molecules is completed very slowly in monocytes, as reverse transcription take place over days after infection. The majority of functional viral genomes are completed by day 4-5 post infection and then 5-6 additional days to integrate (Arfi et al., 2008). This delay in integration of viral DNA into host chromosomal DNA can be due to trafficking, nuclear import, or even postnuclear-import defects specifically present in monocytes. However, these defects delay, but do not diminish, the ability of HIV-1 to infect monocytes.

Overall, the productive infection of monocytes appears to be governed by changes in environmental conditions. Together, the changes in serum concentration, HIV strain (even at low passage), donor source of the monocyte/macrophage and the state of cellular maturation appear to influence HIV replication in monocytes. Thus, it is likely that the host-genetic differences may contribute to differences in HIV replication in monocyte-derived macrophages and consequently to tissue virus load in vivo (Chang et al., 1994). Furthermore, defective immunological function of cells of the macrophage lineage contributes considerably to the pathogenesis of HIV-1 infection. Following HIV-1 infection, effector functions carried out by monocyte/macrophages are also impaired, including phagocytosis, intracellular killing, chemotaxis and cytokine production. Such defects contribute to the pathogenesis of AIDS by allowing reactivation and development of opportunistic infections (Kedzierska et al., 2003a).

Vital contribution of Monocytes in HIV antigen presentation: Monocytes are important in antigen presentation to $\mathrm{T}$ cells during HIV infection. Defective antigen presentation by HIV+monocytes is a problem in HIV isease and its systematic impairment occurs at each stage of plasma viremia. Furthermore, it has been shown that class II expression, formation of class II-Ag complexes and $\mathrm{Ag}$ uptake are impaired in chronically HIV-1-infected monocytic cells, which may contribute to the global immunosuppression observed in AIDS (Polyak et al., 1997). This defective antigen presentation is possibly related to severe immune dysfunction in HIV+patients. The mechanism by which this process occurs is not clear, but it appears that the reduced capacity by HIV-infected monocytes to stimulate or present antigen to CD4+T-cells is mediated by cellular factors associated with the plasma membranes of HIV-infected monocytes, but the soluble factors secreted by HIV-infected monocytes have little or no effect on T-cell stimulation. Thus, the altered capacity of HIV-infected monocytes to stimulate and present antigen to $\mathrm{CD} 4+\mathrm{T}$-cells is related to downmodulation of CD4 expression on T-cells and appears to occur via membrane-associated cellular factors on HIV-infected monocytes (Louie et al., 1996). Another underlying reason could be the induction of TNF by gp120, which may be associated with impairment of antigen-presenting apacity of monocytes seen in AIDS patients (Zembala et al., 1994).

\section{Role of soluble CD14 as a marker and its correlation with HIV disease: $\mathrm{CD} 14$ is a $55-\mathrm{kDa}$ Glycosylphosphatidylinositol (GPI)-linked protein} present on the surface membrane of phagocytic leukocytes. It is also present in a soluble form in serum. Soluble (s) CD14 is a marker for monocyte/macrophage activation and a mediator of bacterial Lipopolysaccharide (LPS) action. The complex glycolipid lipopolysaccharide (LPS), a cell membrane component of gram-negative bacteria, is a potent immune stimulant. This immune stimulation is associated with the binding of this complex with the CD14/Toll-like receptor-4 (TLR-4) complex (Takeuchi et al., 2000). CD14, the primary LPS receptor, exists as both cell surface membrane-bound (mCD14) and soluble forms (sCD14) (Schutt, 1999). mCD14 is expressed on the cell surface of cells from the monocyte/macrophage lineage (Takeuchi et al., 2000) and sCD14 is likely to represent the mCD14 which has been shed in response to either monocyte activation or 
Am. J. Infect. Dis., 8 (2): 92-105, 2012

differentiation (Lynn and Golenbock, 1992). sCD14 can bind to circulating LPS complexes and can activate cells that do not normally express mCD14, such as CD14-monocytes and endothelial cells (Golenbock et al., 1995; Schutt et al., 1995).

In HIV infection, up-regulated expression of mCD14 and increased levels of sCD14 on monocytes (Lien et al., 1998; Nockher et al., 1994) and alveolar macrophages (Wasserman et al., 1994) have been reported. Elevated levels of sCD14 have been shown to correlate with disease progression in cases of HIV/AIDS (Lien et al., 1998; Nockher et al., 1994). Lien et al. (1998) studied serum levels of sCD14 in HIV-1 infected individuals. They found that SCD14 has an inverse correlation to $\mathrm{CD} 4+\mathrm{T}$ cell counts and a highly significant correlation with HIV-1 RNA levels in serum. Thus, sCD14 appears to be associated with advanced HIV disease (Lien et al., 1998) This study showed that CD14 was elevated in serum from HIV-1infected individuals compared with seronegative controls. The highest levels were found in patients with advanced clinical and immunological disease. Patients with ongoing clinical events had significantly higher sCD14 levels than symptomatic HIV-1-infected individuals without clinical events, with especially elevated levels in patients infected with Mycobacterium Avium Complex (MAC). Longitudinal analysis of patients showed that increasing sCD14 serum concentrations per time unit were associated with death. By contrast, this was not the case with monocyte numbers, whereas no differences in $\mathrm{CD} 4+\mathrm{T}$ cell numbers were found between survivors and non-survivors.

The molecular mechanism through which monocyte CD14 expression is up-regulated remains unclear. A recent study undertaken by Creery et al. (2002) showed that the HIV-regulatory protein Nef upregulated $\mathrm{mCD} 14$ expression but fails to induce the release of sCD14. Higher levels of mCD14 and circulating sCD14 in HIV-infected individuals may reflect their chronic state of inflammation and may be involved in enhanced susceptibility to gram-negative infections (Creery et al., 2002).

Recently, it has been shown that in HIV-infected patients, the amounts of MD-2, CD14 and IL-10 increased significantly in the patient group with AIDS. Interesting was that IL-10, CD14 and MD-2 levels were positively correlated, suggesting that IL-10 may be a driving force for increased release of MD-2 and CD14 during systemic inflammation (Sandanger et al., 2009).

Production of cytokines and chemokines by monocytes in HIV-1 infection: HIV infection, at all stages of the disease, is associated with chronic immune activation and dysfunctional cytokine production (Alfano and Poli, 2002; Fantuzzi et al., 2000). Monocytes and macrophages are highly secretory cells and are therefore major contributors to the alteration of the cytokine/chemokine network (Fantuzzi et al., 2003). It is now widely accepted that in HIV-infected individuals there is a decrease in the production of some immuno-regulatory cytokines (interleukin (IL) -2, IL-12, IL-10 and IFN- $\gamma$ ), an increase in proinflammatory cytokines (TNF- $\alpha$, IL-1, IL-6 and IL-8), as well as an increase in production of type 1 interferon's (IFN's) (Gessani et al., 1997). Several reports have shown that monocytes/macrophages produce a variety of cytokines in response to HIV infection (Alfano and Poli, 2002; Amirayan-Chevillard et al., 2000; Gessani et al., 1994). Some of these cytokines have been shown to up-regulate HIV expression, such as TNF- $\alpha$, whereas others have been shown to be powerful inhibitors of HIV replication in macrophages, such as IFN's.

Not only does HIV infection cause a dysfunctional cytokine production, but it also disrupts the normal synthesis of chemokines. A number of studies have demonstrated that exposure of monocytes/macrophages to viral products, such as gp120, can trigger CC chemokine production, as well as through proinflammatory cytokines inducing the production of chemokines such as CCL2 (Badolato et al., 1997). CCL2, CCL3, CCL4 and CCL22 have been consistently detected in monocytes and monocytederived macrophages (Andrew et al., 1998; Bonecchi et al., 1998; Godiska et al., 1997). CC chemokines are responsible for attracting monocytes and leukocytes to sites of infection. When there is an up-regulation of chemokine production it leads to the abnormal movement of monocytes into various different tissue sites. It is this situation that possibly drives monocytes moving through the blood-brain barrier and causing HIV-associated dementia (Yang et al., 2009).

Monocytes-a possible source of HIV during HAART therapy: Recent studies have investigated the levels of cell-associated viral DNA, mRNA and genetic evolution of HIV over the course of infection (Sonza et al., 1996; Zhu, 2002; Zhu et al., 2002). Monocytes isolated from blood have been shown to contain unintegrated circular viral DNA and multiply spliced RNA suggesting their infection is recent and transcriptionally active rather than latent (Sonza et al., 1996). Zhu and colleagues observed that blood-purified monocytes harboured HIV DNA over time in both untreated and therapy-suppressed individuals (Zhu et al., 2002). In a recent study, Potter and Colleagues 
Am. J. Infect. Dis., 8 (2): 92-105, 2012

presented a detailed analysis of HIV-1 populations isolated from total PBMC, plasma, CD4+T cells, $\mathrm{CD} 8+\mathrm{T}$ cells and monocytes/macrophages in 13 patients receiving HAART. Sequence analysis of the reverse transcriptase and protease genes indicated that viral strains isolated from different blood leukocytes were genetically distinct in each subject. Further, the compartmentalization of drug resistance mutations in specific blood cell types was observed in approximately $50 \%$ of patients. The prevalence of resistance mutations was higher in either CD4+T cells or monocytes/macrophages in these subjects. However, $\mathrm{CD} 8+\mathrm{T}$ cells showed markedly lower levels of viral drug resistance in these patients, indicating a lack of viral replication in this compartment. This study is the first to demonstrate the differential distribution of HIV drug resistance in different blood cell types during HAART and provides new insights into the infection of diverse blood leukocytes, including monocytes in vivo (Potter et al., 2003; Saksena and Potter, 2003).

Viral decay in monocytes was considerably slower on average than that in activated and resting CD4+T cells. In addition, the average half-life of HIV DNA in monocytes was considerably longer than the estimated mean inter-mitotic life spans of monocytes and macrophages (Perelson et al., 1996; 1997; Whitelaw and Batho, 1972; Furth, 1989), suggesting renewal of the virus as a result of continued viral replication ( $\mathrm{Zhu}$ et al., 2002). A significant genetic evolution in monocytes was also observed and in some individuals on suppressive therapy monocyte trains were phylogenetically-linked with circulating plasma variants (Zhu et al., 2002). These findings suggest that monocytes may constitute a continuing source of infectious virus during HAART regardless of the length of treatment.

As discussed above, the circulating monocyte population is heterogeneous consisting of several subsets. The majority express high levels of CD14 and little or no CD16, termed as CD14hi. A minor subset representing around $15 \%$ of total circulating monocytes expresses low levels of CD14 and high levels of CD16 (CD14loCD16hi) (Passlick et al., 1989). CD14loCD16hi monocytes may represent a stage in myeloid maturation, either to tissue macrophages or immature dendritic cells. CD14loCD16hi monocytes share some similarities with mature monocytes including their cytokine profiles (Passlick et al., 1989) and are major producers of tumor necrosis factor alpha (TNF- $\alpha$ ) in the blood (Belge et al., 2002). They also share similarities with dendritic cells (Ancuta et al., 2000). The CD14loCD16hi subset expresses elevated levels of CCR5 (Weber et al., 2000) and CD4 (Tanaka et al., 1999) in comparison to CD14hi monocytes. The characteristics of the CD14loCD16hi subset suggest they may be the major targets of HIV. Although the actual source of HIV-1 in these monocytes remains undefined, there is evidence that HIV-1 proviral DNA in monocytes does not originate from myeloid tissue precursors (Spear et al., 1990).

The normal pathways of monocyte migration and trafficking may also define a mechanism by which HIV is distributed to various compartments around the body. After leaving the bone marrow, monocytes remain in the circulation for between two and four days before migrating through the endothelial walls of capillaries (Whitelaw and Batho, 1972) and undergoing differentiation into tissue macrophages. Alternatively they may differentiate into Dendritic Cells (DCs) and enter the lymphatics (Harmsen et al., 1985; Randolph et al., 2002). HIV-infected monocytes can thus migrate to a variety of different sites around the body and are likely to be responsible for colonization and continued turnover in diverse tissue compartments such as the CNS (Nottet et al., 1996; Persidsky et al., 1999). The early establishment of HIV infection in monocytes and the ongoing replication and persistence of HIV in this compartment represents a considerable challenge for antiretroviral drug regimens.

Functional changes in monocytes caused by HIV-1: Although monocytes are not substantially depleted in HIV infection, alterations of function have been recognized for many years. It has been well established that these functional effects play important roles in neurological disease, in AIDS and in defective innate immunity (Collman et al., 2003).

Monocyte differentiation and HIV infection: Bloodcirculating monocytes differentiate in tissues into either macrophages or dendritic cells in both a response to danger stimuli or to replenish resident cells under normal conditions. Due to their migratory behavior and their key functions in immune system responses, it is not surprising that cells of the monocyte-macrophage lineage are often the preferential targets of a number of lentiviruses. Although a vast literature exists on the ability of HIV-1 to replicate in either macrophages or dendritic cells (Collman et al., 2003; Kedzierska et al., 2003b; Verani et al., 2005), blood-circulating monocytes are often considered as an example of a cell type restrictive to HIV-1 infection (Heinzinger et al., 1995; Neil et al., 2001; Sonza et al., 1996). Given that infectious virus can be isolated from monocytes of seropositive patients (Collman et al., 1989), it is clear that monocytes, although resistant, are not completely 
refractory to HIV infection. Thus, cellular differentiation of monocytes to macrophages plays a role in increasing the susceptibility of monocytic cells to viral infection and/or enhancing HIV expression from cells harboring HIV provirus (Goletti et al., 1995). As mentioned below, monocytes up-regulate the expression of TLR's in response to HIV infection. Krutzik et al. (2005) took these findings and explored the role of TLR's in monocyte differentiation. They showed that the activation of TLR's causes the rapid differentiation of monocytes into either DC-SIGN+or CD1b+cells (Krutzik et al., 2005). DC-SIGN+cells have a macrophage-like phenotype, are phagocytic and use DC-SIGN to facilitate the uptake of bacteria. In contrast, CD1b+cells have an immature dendritic cell phenotype, release pro-inflammatory cytokines and function as effective antigen-presenting cells.

Like $\mathrm{T}$ cells, macrophages display immune polarization that can promote or impair adaptive immunity. Fischer-Smith et al. (2008) have hypothesized that dysregulation of monocyte/macrophage activation and differentiation may promote immune dysfunction and contribute to AIDS pathogenesis. Using flow cytometry, they analyzed the frequency of monocyte subsets in HIV-1 infection relative to seronegative controls, focusing on the CD163(+)/CD16(+) monocyte as a likely precursor of the "alternatively activated" macrophage. Individuals with detectable HIV-1 infection showed an increase in the frequency of CD163(+)/CD16(+) monocytes when compared to seronegative or HIV-1-infected persons with undetectable viral loads. A positive correlation was observed between increased CD163(+)/CD16(+) monocyte frequency and plasma viral load. These data suggest a potential role for CD163(+)/CD16(+) monocytes in virus production and disease progression. CD163(+)/CD16(+) monocytes may be a useful biomarker for HIV-1 infection and AIDS progression and a possible target for therapeutic intervention (Fischer-Smith et al., 2008).

Expression of Toll-Like Receptors (TLRs): Receptors of the innate immune system are crucial in the first-line defense against foreign microbes and are important for modulating the adaptive immune response. TLRs represent an important category of such pathogenrecognition receptors. Monocytes have been shown to express TLR-2 and TLR-4 and the stimulation of these receptors induces the activation of $\mathrm{NF \kappa B}$, with subsequent production of inflammatory cytokines and chemokines (Heggelund et al., 2004). Heggelund and his colleagues in 2004 demonstrated that there is an increase in TLR-2 expression on monocytes during HIV infection (Heggelund et al., 2004). After stimulation of Peripheral
Blood Mononuclear Cells (PBMC's) with gp120, there was a significant increase in TLR-2 expression on monocytes, supporting a possible link between HIV infection and TLR-2 expression.

Many studies have shown that ongoing Tumor Necrosis Factor (TNF) production is seen in HIV infected patients even during HAART (Aukrust et al., 1999; Ledru et al., 2000). With the increase of TLR-2 expression on monocytes and the persistent TNF- $\alpha$ presence, it leads to an increase in inflammation effects. Therefore, the increase in TLR-2 expression on monocytes could contribute to HIV-related inflammation.

\section{Relevance of monocytes in HIV disease:}

Viral reservoir: Mononuclear phagocytes (bone marrow monocyte-derived macrophages, alveolar macrophages, perivascular macrophages and microglia) are reservoirs and vehicles of dissemination for HIV-1. Infected monocytes can seed HIV in down-stream lineage cells, namely macrophages, which can then serve as a virus source in multiple tissue sites (Fulcher et al., 2004). Despite whether HIV-1 can replicate in monocytes themselves, once the cell has entered a tissue site and differentiated into either a macrophage or dendritic cell, then the virus can start replicating and infecting the tissue. Tissue macrophages are generally regarded as more important long-lived reservoirs and these cells often support high levels of viral replication. Macrophages have been shown to act as a virus source in non-lymphoid tissues such as the brain, lung, liver and gut (Orenstein et al., 1997). These cellular reservoirs and tissue sanctuary sites allow either continuing lowlevel viral transcription, or are latently infected and contain integrated proviral DNA. Furthermore, all known reverse transcriptase inhibitors are ineffective in chronically infected macrophages, which accentuates the viral persistence in these tissue sites and highlights the importance of this role of monocytes in HIV-1 pathogenesis (Aquaro et al., 1997).

Recently, Gibellini et al. (2008) evaluated HIV-1 DNA loads in Peripheral Blood Leukocytes (PBLs) and monocytes from long-term HAART-treated and antiretroviral naïve HIV-1 infected patients and compared to RNA viral load and CD4+cell count. Their analysis revealed that the HAART-treated patients showed significantly lower levels of viral DNA both in PBLs and monocytes in comparison to therapy naïve individuals. Variable levels of HIV-1 DNA amount in monocytes were detected in all naïve patients but only in 12 of 34 HAART-treated individuals. Their work confirms that the long-term HAART decreased HIV-1 DNA load in PBLs and monocytes demonstrating a valuable inhibitor effect, especially in short-lived 
Am. J. Infect. Dis., 8 (2): 92-105, 2012

reservoirs. In addition, the positive correlation of DNA burden between PBLs and monocytes may suggest a dynamic relation between these reservoirs in the course of disease (Gibellini et al., 2008).

Monocytes and HAART: HIV-1 in blood monocytes can disseminate and evolve independently from CD4+T cells over the course of HIV-1 infection. This presents a major challenge to the treatment of HIV-1 infection because HIV-1 populations in monocytes and macrophages are poorly suppressed, compared to those in $\mathrm{CD} 4+\mathrm{T}$ cells and replicate relatively actively in patients undergoing seemingly effective HAART (Fulcher et al., 2004). Zhu et al. (2002) showed that HIV-1 can replicate in CD14+monocytes in vivo, even in patients receiving HAART (Zhu et al., 2002). Monocytes/macrophages can maintain HIV-1 replication even throughout HAART, because the antiretroviral drugs may not block viral replication in these cells as efficiently as in CD4+T cells. This is because cells from the monocyte/macrophage lineage have quite different characteristics to lymphocytes, in terms of cellular metabolism, membrane receptors and responsiveness to cytokines (Aquaro et al., 1997).

Aquaro et al. (1997) performed a comprehensive assessment of the effects of most antiretroviral drugs on both lymphocytes and monocyte/macrophages. They found that all clinically relevant nucleoside analogue inhibitors of reverse transcriptase were more effective in monocyte/macrophages then in lymphocytes. However, these drugs have limited effect against HIVrelated encephalopathy because of the poor penetration of these drugs into the central nervous system, rather than their limited effect on HIV-infected monocytes/macrophages. Protease inhibitors were found to be the only drug able to affect the replication of HIV in monocytes/macrophages already carrying the proviral genome. However, it is unknown as to whether protease inhibitors in plasma and issues maintain high enough concentrations to be active on chronically infected cells (Aquaro et al., 1997). Therefore, future drugs targeting monocytes/macrophages should be aimed at the later stages of HIV life cycle.

Monocytes supporting HIV persistence in vivo: HIV1 is often continually present in the body despite ongoing HAART therapy. Three models have been proposed to account for HIV-1 persistence in relation to monocytes. The first model is based on monocytes serving as a direct source of plasma virus by producing infectious HIV-1 in peripheral blood (Zhu, 2002). In patients without antiretroviral therapy, activated CD4+T cells may produce up to $99 \%$ of all virus particles while the other $1 \%$ of virus may be generated primarily from tissue macrophages. In this situation, viruses produced by blood monocytes may be too minor to recognize. However, in patients on suppressive HAART in whom HIV replication in activated CD4+T cells is blocked, viruses produced from monocytes become relatively dominant. Zhu (2002) showed that there was a higher level of HIV-1 replication in CD14+monocytes compared with resting CD4+T cells in patients undergoing HAART (Zhu, 2002). This demonstrates that monocytes can contribute to plasma virus and therefore contribute to HIV persistence within the infected individual.

The second model to account for the persistence of HIV-1 in blood monocytes is that HIV-infected precursor cells of monocytes in bone marrow enter and renew HIV-1 infected monocytes in peripheral blood. These monocyte precursor cells, namely CD34+progenitor cells, may be infected with HIV, then enter the bloodstream and renew the viral pool in peripheral blood monocytes. However, there is much controversy in this area of investigation, as it is still unknown as to whether CD34+progenitor cells are susceptible to HIV-1 infection (Alexaki and Wigdahl, 2008).

The third model for HIV-1 persistence is that HIVinfected monocytes can act as a viral reservoir or viral source, as discussed previously. These viral sources maintain HIV-1 in sites most often inaccessible to HAART and contribute to HIV-persistence.

HIV Neuropathogenesis: Monocyte-macrophage trafficking through the blood brain barrier: An interesting aspect of the previously mentioned role of monocytes as viral reservoirs is in neuropathogenesis related to HIV. HIV-1 enters the brain during the establishment and systemic dissemination of HIV-1 infection and causes a broad range of HIV-1 associated neurocognitive disorders, including HIV-1-Associated Dementia (HAD) in at least $30 \%$ of HIV patients. Even in the era of HAART, HAD continues to be a problem because of suboptimal concentrations of drugs reaching the CNS. HAD is a devastating neurological disease which is characterized by typical pathological changes in the brain and spinal cord. HIV-induced changes in the brain are most evident in the white matter and they include gross cerebral atrophy without inflammation, but associated with microglial nodules (clusters of microglia and reactive fibrous astrocytes) and multinucleated giant cells (Meltzer et al., 1990).

There is now general agreement that the cells supporting productive infection in brain are the 
microglial cells and macrophages, whereas the neurons and oligodendrocytes are relatively rarely infected (Bagasra et al., 1996). The route of CNS infection appears to involve Circulating Activated Monocytes (CAM), which increase in proportion as the disease stage of an individual progress (Gartner, 2000; Pulliam et al., 1997). The peripheral activation of circulating monocytes is the critical step for viral entry into the CNS (Gartner, 2000). It has been shown that individuals seropositive for HIV may contain little or no DNA, despite early entry of HIV into the brain (Donaldson et al., 1994). Even if DNA is evident, there is no expression of HIV structural proteins (Kibayashi $e t$ al., 1996). Thus, the reseeding of the CNS by activated monocytes leading to productive CNS infection remains the only plausible mechanism of viral entry into the brain, which, in most cases, does not occur until an advanced stage of HIV disease (Kim et al., 2003).

Further, macrophage activation within the CNS and Peripheral Nervous System (PNS) appears to be a critical factor in the development of HIV-D and sensory neuropathies (Keswani et al., 2002). Since the emergence of a subset of circulating monocytes during HIV-1 disease appears to correlate with cognitive impairment, it has been hypothesized that diagnostic protein profiles may be obtained from this monocytic subset especially for patient at risk for HIV-D. Wojna et al. (2004) with the help of sophisticated proteomic techniques (surface enhance laser desorption/ionizationtime of flight protein chip assay) have elegantly shown with a case study seven unique proteins between 3 and $20 \mathrm{kD}$ in Monocyte-derived Macrophges (MDM) from patients with HIV Associated Dementia (HAD), which were absent in the control group. Further, all these proteins were abrogated after HAART. Recently, (Sun et al., 2004) have also shown that there is loss of macrophage-secreted lyzozyme in HIV-D as shown by SELDI-TOF mass spectrometry. Thus, both studies confirm macrophage dysfunction as a significant consequence to HAD and both emphasize the utility of MDM profiling for the diagnosis and monitoring of HIV-D.

Viral inflammation and replication: Monocytes play a crucial role in the early innate immunity response to all infections. They recognize non-self molecules and through the release of cytokines and chemokines they can stimulate other immune cells, as well as the adaptive immune response, to clear the infection. These cytokines and chemokines can also affect viral replication and even pathogenesis, however the contribution of monocytes to viral replication remains unclear. Kuwata et al. (2007) showed that monocyte cell count has a close relation to viral load. This suggests that monocytes have significant effects on viral replication in vivo and that monocytes may play a role in controlling viral replication in the early phase of infection (Kuwata et al., 2007). In the later stages of disease, monocytes may not have such an effect on viral replication or progression of disease because in this phase adaptive immunity plays the main role.

Recently, interest has grown around whether monocytes can harbor replicative-petent HIV-1. A study by Crowe et al. (2003) showed that monocytes contain HIV-1 DNA in both untreated and treated patients. Furthermore, viral decay in monocytes was slower on average than that in activated and resting CD4+T cells and the mean half-life of HIV-1 DNA in monocytes was longer then that in resting and activated CD4+T cells (Crowe et al., 2003). This suggests that either the blood monocytes are recently infected, or there is ongoing viral replication in monocytes or in its precursor cells. This finding is controversial, as there have also been a number of studies that have showed that monocytes are unable to release HIV-1 virions.

\section{CD14+monocyte transcriptome and its subversion} by HIV: There has been much interest surrounding the role that monocytes play in HIV-1 pathogenesis because of their seemingly crucial role in combating the infection. One recent method for studying these cells is by analyzing their gene expression at different stages of HIV-1 disease. This form of method hopes to reveal important genes or proteins that will define the role that monocytes play in HIV-1 pathogenesis and gain to our understanding of HIV-1 to hopefully create more efficient treatment regimes. Giri and his colleagues performed one such study in early 2009, where they analyzed the gene expression of circulating monocytes in HIV-1 infected subjects. They studied HIV+ndividuals with viral loads of between 3000 and 100000 copies $\mathrm{ml}^{-1}$ of plasma and who were untreated patients. After microarray analyses, there were a number of genes found to be differentially regulated, but they chose to focus on apoptosis-related genes because they were significantly over-represented by functional group clustering analysis. In this group there were 58 genes in total and 38 of these genes have been directly reported as having pro-and anti-apoptotic function. Of these 38 genes, 28 of them were stably modulated to suggest increased monocyte survival, i.e., pro-apoptotic genes were down regulated, or antiapoptotic genes were up regulated. Through functional analysis, Giri and her colleagues also showed that these 
genes are associated with four pathways, being p53 modulation, TNF signaling, CD40L signaling and MAPK signaling (Giri et al., 2009).

Giri and her colleagues were the first to provide evidence of a stable anti-apoptosis gene expression in monocytes in chronic HIV-1 infection. However, this study only tested 14,000 genes from the human genome. There have been no studies performed on monocytes in HIV-1+patients, 1 which encompass all 25,000 human genes, therefore the studies by Giri et al. (2009) are preliminary.

Monocyte-derived macrophage secretome: Very little appears to have been explored at the level of monocyte proteome in the context of HIV infection. To understand HIV and its interaction with monocytes, a clear understanding of correlation between trancriptome and proteome is necessary in order to validate if some transcripts are also expressed at the protein level. This can further provide functional validation and identify protein candidates, which may possibly be involved in HIV pathogenesis of monocytes. Recently, Ciborowski et al. (2007) analyzed the secretome of HIV-1-infected human monocyte-derived macrophages. They identified 110 proteins in culture supernatants of control (uninfected) and virus-infected cells. Differentially expressed cytoskeletal, enzymes, redox and immunoregulatory protein classes were discovered, which were validated by Western blot tests. These included cystatin $\mathrm{C}$, cystatin $\mathrm{B}$, chitinase 3-like 1 protein, cofilin-1, 1-plastin, superoxide dismutase, leukotrieneA(4) hydrolase and alpha-enolase. Although this study, using high throughput proteomic approach, provides insights into virus-host cell interactions that likely affect the functional role of monocyte-derived macrophages in HIV disease, detailed studies are needed to validate these data on primary monocytes from HIV patients at different stages of HIV disease, which may provide a snapshot of proteins relevant in vivo. The studies by Ciprowski will provide a good comparison against primary monocytes.

\section{CONCLUSION}

The functional improvement of monocytes, which are one of the critical components of the innate antimicrobial immunity, is likely to contribute to the improved cell-mediated immunity against opportunistic infections in HIV patients receiving HAART. More work is needed at the cell subset level to define which monocytic subsets are impaired during HIV viremia, so that functional restoration can be achieved through appropriate interventions in HIV patients to improve cell-mediated immune responses.

\section{REFERENCES}

Alexaki, A. and B. Wigdahl, 2008. HIV-1 infection of bone marrow hematopoietic progenitor cells and their role in trafficking and viral dissemination. PLoS Pathog., 4: e1000215-e1000215. PMID: 19112504

Alfano, M. and G. Poli, 2002. The cytokine network in HIV infection. Curr. Mol. Med., 2: 677-689. PMID: 12462389

Amirayan-Chevillard, N., H. Tissot-Dupont, C. Capo, C. Brunet and F. Dignat-George et al., 2000. Impact of highly active anti-retroviral therapy (HAART) on cytokine production and monocyte subsets in HIV-infected patients. Clin. Exp. Immunol., 120: 107-112. PMID: 10759771

Ancuta, P., L. Weiss and N. Haeffner-Cavaillon, 2000. CD14+CD16++ cells derived in vitro from peripheral blood monocytes exhibit phenotypic and functional dendritic cell-like characteristics. Eur. J. Immunol., 30: 1872-1883. PMID: 10940876

Andrew, D.P., M.S. Chang, J. McNinch, S.T. Wathen and M. Rihanek et al., 1998. STCP-1 (MDC) CC chemokine acts specifically on chronically activated Th2 lymphocytes and is produced by monocytes on stimulation with Th2 cytokines IL-4 and IL-13. J. Immunol., 161: 5027-5038. PMID: 9794440

Aquaro, S., C.F. Perno, E. Balestra, J. Balzarini and A. Cenci et al., 1997. Inhibition of replication of HIV in primary monocyte/macrophages by different antiviral drugs and comparative efficacy in lymphocytes. J. Leukoc. Biol., 62: 138-143. PMID: 9226005

Arfi, V., L. Riviere, L. Jarrosson-Wuilleme, C. Goujon and D. Rigal et al., 2008. Characterization of the early steps of infection of primary blood monocytes by human immunodeficiency virus type 1. J. Virol., 82: 6557-6565. PMID: 18417568

Aukrust, P., F. Muller, E. Lien, I. Nordoy and N.B. Liabakk et al., 1999. Tumor Necrosis Factor (TNF) system levels in human immunodeficiency virusinfected patients during highly active antiretroviral therapy: Persistent TNF activation is associated with virologic and immunologic treatment failure. J. Infect. Dis., 179: 74-82. PMID: 9841825

Badolato, R., A.N. Ponzi, M. Millesimo, L.D. Notarangelo and T. Musso, 1997. Interleukin-15 (IL-15) induces IL-8 and monocyte chemotactic protein 1 production in human monocytes. Blood, 90: 2804-2809. PMID: 9326248 
Bagasra, O., E. Lavi, L. Bobroski, K. Khalili and J.P. Pestaner et al., 1996. Cellular reservoirs of HIV-1 in the central nervous system of infected individuals: Identification by the combination of in situ polymerase chain reaction and immunohistochemistry. AIDS, 10: 573-585. PMID: 8780811

Barre-Sinoussi, F., J.C. Chermann, F. Rey, M.T. Nugeyre and S. Chamaret et al., 1983. Isolation of a T-lymphotropic retrovirus from a patient at risk for Acquired Immune Deficiency Syndrome (AIDS). Science, 220: 868-871. PMID: 6189183

Belge, K.U., F. Dayyani, A. Horelt, M. Siedlar and M. Frankenberger et al., 2002. The proinflammatory CD14+CD16+DR++ monocytes are a major source of TNF. J. Immunol., 168: 3536-3542. PMID: 11907116

Bonecchi, R., S. Sozzani, J.T. Stine, W. Luini and G. D'Amico et al., 1998. Divergent effects of interleukin-4 and interferon-gamma on macrophage-derived chemokine production: An amplification circuit of polarized $\mathrm{T}$ helper 2 responses. Blood, 92: 2668-2671. PMID: 9763548

Chang, J., S. Li, H. Naif and A.L. Cunningham, 1994. The magnitude of HIV replication in monocytes and macrophages is influenced by environmental conditions, viral strain and host cells. J. Leukoc. Biol., 56: 230-235. PMID: 8083594

Ciborowski, P., I. Kadiu, W. Rozek, L. Smith and K. Bernhardt et al., 2007. Investigating the human immunodeficiency virus type 1-infected monocytederived macrophage secretome. Virology, 363: 198-209. PMID: 17320137

Clavel, F., D. Guetard, F. Brun-Vezinet, S. Chamaret and M.A. Rey et al., 1986. Isolation of a new human retrovirus from West African patients with AIDS. Science, 233: 343-346. DOI: $10.1126 /$ science. 2425430

Coffin, J., A. Haase, J.A. Levy, L. Montagnier and S. Oroszlan et al., 1986. What to call the AIDS virus? Nature, 321: 10-10. PMID: 3010128

Collman, R., N.F. Hassan, R. Walker, B. Godfrey and J. Cutilli et al., 1989. Infection of monocyte-derived macrophages with Human Immunodeficiency Virus type 1 (HIV-1). Monocyte-tropic and lymphocyte-tropic strains of HIV-1 show distinctive patterns of replication in a panel of cell types. J. Exp. Med., 170: 1149-1163. PMID: 2571666

Collman, R.G., C.F. Perno, S.M. Crowe, M. Stevenson and L.J. Montaner, 2003. HIV and cells of macrophage/dendritic lineage and other non-T cell reservoirs: New answers yield new questions. J. Leukoc. Biol., 74: 631-634. PMID: 12960251
Creery, D., J.B. Angel, S. Aucoin, W. Weiss and W.D. Cameron et al., 2002. Nef protein of human immunodeficiency virus and lipopolysaccharide induce expression of CD14 on human monocytes through differential utilization of interleukin-10. Clin. Diagn. Lab. Immunol., 9: 1212-1221. PMID: 12414752

Crowe, S., T. Zhu and W.A. Muller, 2003. The contribution of monocyte infection and trafficking to viralpersistence and maintenance of the viral reservoir in HIV infection. J. Leukoc. Biol., 74: 635-641. PMID: 12960232

Donaldson, Y.K., J.E. Bell, J.W. Ironside, R.P. Brettle and J.R. Robertson et al., 1994. Redistribution of HIV outside the lymphoid system with onset of AIDS Lancet, 343: 383-385. PMID: 7905551

Fantuzzi, L., F. Belardelli and S. Gessani, 2003. Monocyte/macrophage-derived $\mathrm{CC}$ chemokines and their modulation by HIV-1 and cytokines: A complex network of interactions influencing viral replication and AIDS pathogenesis. J. Leukoc. Biol., 74: 719-725. PMID: 12960239

Fantuzzi, L., L. Conti, M.C. Gauzzi, P. Eid and M. Del Corno et al., 2000. Regulation of chemokine/cytokine network during in vitro differentiation and HIV-1 infection of human monocytes: Possible importance in the pathogenesis of AIDS. J. Leukoc. Biol., 68: 391399. PMID: 10985256

Fischer-Smith, T., E.M. Tedaldi and J. Rappaport, 2008. CD163/CD16 coexpression by circulating monocytes/macrophages in HIV: Potential biomarkers for HIV infection and AIDS progression. AIDS Res. Hum. Retroviruses, 24: 417-421. PMID: 18373432

Fulcher, J.A., Y. Hwangbo, R. Zioni, D. Nickle and X. Lin et al., 2004. Compartmentalization of human immunodeficiency virus type 1 between blood monocytes and CD4+T cells during infection. J. Virol., 78: 7883-7893. PMID: 15254161

Furth, R.V., 1989. Origin and turnover of monocytes and macrophages. Curr. Top Pathol., 79: 125-150. PMID: 2644082

Gartner, S., 2000. HIV infection and dementia. Science, 287: 602-604. PMID: 10691542

Gartner, S., P. Markovits, D.M. Markovitz, R.F. Betts and M. Popovic, 1986. Virus isolation from and identification of HTLV-III/LAV-producing cells in brain tissue from a patient with AIDS. JAMA, 256: 2365-2371. PMID: 3490587 
Gessani, S., P. Borghi, L. Fantuzzi, B. Varano and L. Conti et al., 1997. Induction of cytokines by HIV-1 and its gp120 protein in human peripheral blood monocyte/macrophages and modulation of cytokine response during differentiation. J. Leukoc. Biol., 62: 49-53. PMID: 9225992

Gessani, S., P. Puddu, B. Varano, P. Borghi and L. Conti et al., 1994. Induction of beta interferon by human immunodeficiency virus type 1 and its gp120 protein in human monocytes-macrophages: role of beta interferon in restriction of virus replication. J. Virol., 68: 1983-1986. PMID: 8107259

Gibellini, D., M. Borderi, E. De Crignis, R. Cicola and L. Cimatti et al., 2008. HIV-1 DNA load analysis in peripheral blood lymphocytes and monocytes from naïve and HAART-treated individuals. J. Infect., 56: 219-225. PMID: 18276011

Giri, M.S., M. Nebozyhn, A. Raymond, B. Gekonge and A. Hancock et al., 2009. Circulating monocytes in HIV-1-infected viremic subjects exhibit an antiapoptosis gene signature and virusand host-mediated apoptosis resistance. J. Immunol., 182: 4459-4470. PMID: 19299747

Godiska, R., D. Chantry, C.J. Raport, S. Sozzani and P. Allavena et al., 1997. Human Macrophage-derived Chemokine (MDC), a novel chemoattractant for monocytes, monocyte-derived dendritic cells and natural killer cells. J. Exp. Med., 185: 1595-1604. DOI: $10.1084 /$ jem.185.9.1595

Golenbock, D.T., R.R. Bach, H. Lichenstein, T.S. Juan and A. Tadavarthy et al., 1995. Soluble CD14 promotes LPS activation of CD14-deficient PNH monocytes and endothelial cells. J. Lab. Clin. Med., 125: 662-671. PMID: 7537790

Goletti, D., A.L. Kinter, P. Biswas, S.M. Bende and G. Poli et al., 1995. Effect of cellular differentiation on cytokine-induced expression of human immunodeficiency virus in chronically infected promonocytic cells: Dissociation of cellular differentiation and viral expression. J. Virol., 69: 2540-2546. PMID: 7884904

Harmsen, A.G., B.A. Muggenburg, M.B. Snipes and D.E. Bice, 1985. The role of macrophages in particle translocation from lungs to lymph nodes. Science, 230: 1277-1280. PMID: 4071052

Heggelund, L., F. Muller, E. Lien, A. Yndestad and T. Ueland et al., 2004. Increased expression of tolllike receptor 2 on monocytes in HIV infection: Possible roles in inflammation and viral replication. Clin. Infect. Dis., 39: 264-269. PMID: 15307037
Heinzinger, N., L. Baca-Regen, M. Stevenson and H.E. Gendelman, 1995. Efficient synthesis of viral nucleic acids following monocyte infection by HIV-1. Virology, 206: 731-735. PMID: 7831833

Ho, P.C., W.K. So and H.K. Ma, 1986. Obstetric performance of elderly primiparae. Asia Oceania. J. Obstet. Gynaecol., 12: 499-504. PMID: 3827729

Innocenti, P., M. Ottmann, P. Morand, P. Leclercq and J.M. Seigneurin, 1992. HIV-1 in blood monocytes: Frequency of detection of proviral DNA using PCR and comparison with the total CD4 count. AIDS Res. Hum. Retroviruses, 8: 261-268. PMID: 1347227

Jolly, C., K. Kashefi, M. Hollinshead and Q.J. Sattentau, 2004. HIV-1 cell to cell transfer across an Env-induced, actin-dependent synapse. J. Exp. Med., 199: 283-293. PMID: 14734528

Joly, M. and J.M. Pinto, 2005. CXCR4 and CCR5 regulation and expression patterns on $\mathrm{T}$-and monocyte-acrophage cell lineages: Implications for susceptibility to infection by HIV-1. Math Biosci., 195: 92-126. PMID: 15893340

Kedzierska, K., R. Azzam, P. Ellery, J. Mak and A. Jaworowski et al., 2003a. Defective phagocytosis by human monocyte/macrophages following HIV1 infection: Underlying mechanisms and modulation by adjunctive cytokine therapy. J. Clin. Virol., 26: 247-263. PMID: 12600656

Kedzierska, K., S.M. Crowe, S. Turville and A.L. Cunningham, 2003b. The influence of cytokines, chemokines and their receptors on HIV-1 replication in monocytes and macrophages. Rev. Med. Virol., 13: 39-56. PMID: 12516061

Keswani, S.C., C.A. Pardo, C.L. Cherry, A. Hoke and J.C. McArthur, 2002. HIV-associated sensory neuropathies. AIDS, 16: 2105-2117. PMID: 12409731

Kibayashi, K., A.R. Mastri and C.S. Hirsch, 1996. Neuropathology of human immunodeficiency virus infection at different disease stages. Hum. Pathol., 27: 637-642. PMID: 8698305

Kim, W.K., S. Corey, X. Alvarez and K. Williams, 2003. Monocyte/macrophage traffic in HIV and SIV encephalitis. J. Leukoc. Biol., 74: 650-656. PMID: 12960230

Klatzmann, D., E . Champagne, S. Chamaret, J. Gruest and D. Guetard et al., 1984. T-lymphocyte T4 molecule behaves as the receptor for human retrovirus LAV. Nature, 312: 767-768. PMID: 6083454 
Krutzik, S.R., B. Tan, H. Li, M.T. Ochoa and P.T. Liu et al., 2005. TLR activation triggers the rapid differentiation of monocytes into macrophages and dendritic cells. Nat. Med., 11: 653-660. PMID: 15880118

Kuwata, T., M. Kodama, A. Sato, H. Suzuki and Y. Miyazaki et al., 2007. Contribution of monocytes to viral replication in macaques during acute infection with simian immunodeficiency virus. AIDS Res. Hum. Retroviruses, 23: 372-380. PMID: 17411370

Lambotte, O., Y. Taoufik, M.G.D. Goer, C. Wallon and C. Goujard et al., 2000. Detection of infectious HIV in circulating monocytes from patients on prolonged highly active antiretroviral therapy. J. Acquir. Immune. Defic. Syndr., 23: 114-119. PMID: 10737425

Ledru, E., N. Christeff, O. Patey, P. de Truchis and J.C. Melchior et al., 2000. Alteration of tumor necrosis factor-alpha T-cell homeostasis following potent antiretroviral therapy: Contribution to the development of human immunodeficiency virusassociated lipodystrophy syndrome. Blood, 95: 3191-3198. PMID: 10807787

Lien, E., P. Aukrust, A. Sundan, F. Muller and S.S. Froland et al., 1998. Elevated levels of serumsoluble CD14 in human immunodeficiency virus type 1 (HIV-1) infection: Correlation to disease progression and clinical events. Blood, 92: 20842092. PMID: 9731066

Livingstone, W.J., M. Moore, D. Innes, J.E. Bell and P. Simmonds, 1996. Frequent infection of peripheral blood CD8-positive T-lymphocytes with HIV-1. Edinburgh Heterosexual Transmission Study Group. Lancet, 348: 649-654. PMID: 8782755

Louie, A.T., L.M. Wahl, I.K. Hewlett, J.S. Epstein and S. Dhawan, 1996. Impaired antigen presentation to CD4+T-cells by HIV-infected monocytes is related to down-modulation of CD4 expression on helper T-cells: Possible involvement of HIV-induced cellular factors. FEBS Lett., 398: 1-6. PMID: 8946943

Lynn, W.A. and D.T. Golenbock, 1992. Lipopolysaccharide antagonists. Immunol. Today, 13: 271-276. PMID: 1388655

McElrath, M.J., J.E. Pruett and Z.A. Cohn, 1989. Mononuclear phagocytes of blood and bone marrow: comparative roles as viral reservoirs in human immunodeficiency virus type 1 infections. Proc. Natl. Acad Sci. USA, 86: 675-679. PMID: 2492110
McElrath, M.J., R.M. Steinman and Z.A. Cohn, 1991. Latent HIV-1 infection in enriched populations of blood monocytes and $\mathrm{T}$ cells from seropositive patients. J. Clin. Invest., 87: 27-30. PMID: 1985101

Meltzer, M.S., D.R. Skillman, P.J. Gomatos, D.C. Kalter and H.E. Gendelman, 1990. Role of mononuclear phagocytes in the pathogenesis of human immunodeficiency virus infection. Annu. Rev. Immunol., 8: 169-194. PMID: 2188662

Naif, H.M., S. Li, M. Alali, A. Sloane and L. Wu et al., 1998. CCR5 expression correlates with susceptibility of maturing monocytes to human immunodeficiency virus type 1 infection. J. Virol., 72: 830-836. PMID: 9420295

Neil, S., F. Martin, Y. Ikeda and M. Collins, 2001. Postentry restriction to human immunodeficiency virus-based vector transduction in human monocytes. J. Virol., 75: 5448-5456. PMID: 11356951

Nockher, W.A., L. Bergmann and J.E. Scherberich, 1994. Increased soluble CD14 serum levels and altered CD14 expression of peripheral blood monocytes in HIV-infected patients. Clin. Exp. Immunol., 98: 369-374. PMID: 7527738

Nottet, H.S., Y. Persidsky, V.G. Sasseville, A.N. Nukuna and P. Bock et al., 1996. Mechanisms for the transendothelial migration of HIV-1-infected monocytes into brain. J. Immunol., 156: 12841295. PMID: 8558009

Orenstein, J.M., C. Fox and S.M. Wahl, 1997. Macrophages as a source of HIV during opportunistic infections. Science, 276: 1857-1861. DOI: $10.1126 /$ science.276.5320.1857

Passlick, B., D. Flieger and H.W. Ziegler-Heitbrock, 1989. Identification and characterization of a novel monocyte subpopulation in human peripheral blood. Blood, 74: 2527-2534. PMID: 2478233

Patterson, B.K., D.J. Carlo, M.H. Kaplan, M. Marecki and S. Pawha et al., 1999. Cell-associated HIV-1 messenger RNA and DNA in T-helper cell and monocytes in asymptomatic HIV-1-infected subjects on HAART plus an inactivated HIV-1 immunogen. AIDS, 13: 1607-1611. PMID: 10509560

Patterson, B.K., M. Till, P. Otto, C. Goolsby and M.R. Furtado et al., 1993. Detection of HIV-1 DNA and messenger RNA in individual cells by PCR-driven in situ hybridization and flow cytometry. Science, 260: 976-979. PMID: 8493534 
Patterson, S., M.S. Roberts, N.R. English, A. Stackpoole and M.N. Gompels et al., 1995. HIV infection of blood dendritic cells in vitro and in vivo. Adv. Exp. Med. Biol., 378: 443-445.

Perelson, A.S., A.U. Neumann, M. Markowitz, J.M. Leonard and D.D. Ho, 1996. HIV-1 dynamics in vivo: Virion clearance rate, infected cell life-span and viral generation time. Science, 271: 15821586. PMID: 8599114

Perelson, A.S., P. Essunger, Y. Cao, M. Vesanen and A. Hurley et al., 1997. Decay characteristics of HIV-1-infected compartments during combination therapy. Nature, 387: 188-191. PMID: 9144290

Persidsky, Y., A. Ghorpade, J. Rasmussen, J. Limoges and X.J. Liu et al., 1999. Microglial and astrocyte chemokines regulate monocyte migration through the blood-brain barrier in human immunodeficiency virus-1 encephalitis. Am. J. Pathol., 155: 1599-1611. PMID: 10550317

Polyak, S., H. Chen, D. Hirsch, I. George and R. Hershberg et al., 1997. Impaired class II expression and antigen uptake in monocytic cells after HIV-1 infection. J. Immunol., 159: 2177-2188. PMID: 9278305

Potter, S.J., D.E. Dwyer and N.K. Saksena, 2003. Differential cellular distribution of HIV-1 drug resistance in vivo: Evidence for infection of CD8+T cells during HAART. Virology, 305: 339352. PMID: 12573579

Pulliam, L., R. Gascon, M. Stubblebine, D. McGuire and M.S. McGrath, 1997. Unique monocyte subset in patients with AIDS dementia. Lancet, 49: 692695. PMID: 9078201

Randolph, G.J., G. Sanchez-Schmitz, R.M. Liebman and K. Schakel, 2002. The CD16(+) (FcgammaRIII(+)) subset of human monocytes preferentially becomes migratory dendritic cells in a model tissue setting. J. Exp. Med., 196: 517-527. PMID: 12186843

Rowland-Jones, S.L. and H.C. Whittle, 2007. Out of Africa: What can we learn from HIV-2 about protective immunity to HIV-1? Nat. Immunol., 8: 329-331. DOI:10.1038/ni0407-329

Saksena, N.K. and S.J. Potter, 2003. Reservoirs of HIV1 in vivo: Implications for antiretroviral therapy. AIDS Rev., 5: 3-18. PMID: 12875103

Sandanger, O., L. Ryan, J. Bohnhorst, A.C. Iversen and H. Husebye et al., 2009. IL-10 enhances MD-2 and CD14 expression in monocytes and the proteins are increased and correlated in HIV-infected patients. J. Immunol., 182: 588-595. PMID: 19109192
Schnittman, S.M., M.C. Psallidopoulos, H.C. Lane, L. Thompson and M. Baseler et al., 1989. The reservoir for HIV-1 in human peripheral blood is a $\mathrm{T}$ cell that maintains expression of CD4. Science, 245: 305-308. PMID: 2665081

Schutt, C., 1999. Fighting infection: The role of lipopolysaccharide binding proteins CD14 and LBP. Pathobiology, 67: 227-229. PMID: 10725789

Schutt, C., T. Schilling, U. Grunwald, F. Stelter and S. Witt et al., 1995. Human monocytes lacking the membrane-bound form of the bacterial Lipopolysaccharide (LPS) receptor CD14 can mount an LPS-nduced oxidative burst response mediated by a soluble form of CD14. Res. Immunol., 146: 339-350. PMID: 8719658

Sonza, S., A. Maerz, N. Deacon, J. Meanger, J. Mills and S. Crowe, 1996. Human immunodeficiency virus type 1 replication is blocked prior to reverse transcription and integration in freshly isolated peripheral blood monocytes. J. Virol., 70: 38633869. PMID: 8648722

Spear, G.T., C.Y. Ou, H.A. Kessler, J.L. Moore and G. Schochetman et al., 1990. Analysis of lymphocytes, monocytes and neutrophils from Human Immunodeficiency Virus (HIV)-infected persons for HIV DNA. J. Infect. Dis., 162: 12391244. PMID: 1977808

Sun, B., H.C. Rempel and L. Pulliam, 2004. Loss of macrophage-secreted lysozyme in HIV-1associated dementia detected by SELDI-TOF mass spectrometry. AIDS, 18: 1009-1012. PMID: 15096803

Swirski, F.K., M. Nahrendorf, M. Etzrodt, M. Wildgruber and V. Cortez-Retamozo et al., 2009. Identification of splenic reservoir monocytes and their deployment to inflammatory sites. Science, 325: 612-616. DOI: 10.1126/science. 1175202

Takeuchi, O., K. Hoshino and S. Akira, 2000. Cutting edge: LR2-deficient and MyD88-deficient mice are highly susceptible to Staphylococcus aureus infection. J. Immunol., 165: 5392-5396. PMID: 11067888

Tanaka, Y., M. Kameoka, K. Ota, A. Itaya and K. Ikuta et al., 1999. Establishment of persistent infection with HIV-1 abrogates the caspase-3-dependent apoptotic signaling pathway in U937 cells. Exp. Cell Res., 247: 514-524. PMID: 10066379

Tuttle, D.L., J.K. Harrison, C. Anders, J.W. Sleasman and M.M. Goodenow, 1998. Expression of CCR5 increases during monocyte differentiation and directly mediates macrophage susceptibility to infection by human immunodeficiency virus type 1. J. Virol., 72: 4962-4969. PMID: 9573265 
Verani, A., G. Gras and G. Pancino, 2005. Macrophages and HIV-1: Dangerous liaisons. Mol. Immunol., 42: 195-12. PMID: 15488608

Wasserman, K., M. Subklewe, G. Pothoff, N. Banik and E. Schell-Frederick, 1994. Expression of surface markers on alveolar macrophages from symptomatic patients with HIV infection as detected by flow cytometry. Chest, 1051: 13241334. PMID: 8181314

Weber, K.T., D. Hammache, J. Fantini and B. Ganem, 2000. Synthesis of glycolipid analogues that disrupt binding of HIV-1 gp120 to galactosylceramide. Bioorg. Med. Chem. Lett., 10: 1011-1014. PMID: 10843204

Whitelaw, D.M. and H.F. Batho, 1972. The distribution of monocytes in the rat. Cell Tissue Kinet, 5: 215225. PMID: 5025656

Wojna, V., K.A. Carlson, X. Luo, R. Mayo and L.M. Melendez et al., 2004. Proteomic fingerprinting of human immunodeficiency virus type 1 -associated dementia from patient monocyte-erived macrophages: A case study. J. Neurovirol., 10: 7481. PMID: 14982743
Yang, B., S. Akhter, A. Chaudhuri and G.D. Kanmogne, 2009. HIV-1 gp120 induces cytokine expression, leukocyte adhesion and transmigration across the blood-brain barrier: Modulatory effects of STAT1 signaling. Microvasc. Res., 77: 212-219. PMID: 19103208

Zembala, M., J. Pryjma, A. Plucienniczak, A. Szczepanek and I. Ruggiero et al., 1994. Modulation of antigen-presenting capacity of human monocytes by HIV-1 GP120 molecule fragments. Immunol. Invest., 23: 189-199. PMID: 8070847

Zhu, T., 2002. HIV-1 in peripheral blood monocytes: an underrated viral source. J. Antimicrob. Chemother., 50: 309-311. PMID: 12205054

Zhu, T., D. Muthui, S. Holte, D. Nickle and F. Feng et al., 2002. Evidence for human immunodeficiency virus type 1 replication in vivo in CD14(+) monocytes and its potential role as a source of virus in patients on highly active antiretroviral therapy. J. Virol., 76: 707-716. PMID: 11752161

Ziegler-Heitbrock, L., 2007. The CD14+CD16+blood monocytes: Their role in infection and inflammation. J. Leukoc. Biol., 81: 584-592. PMID: 17135573 